\title{
Heparin bridge is associated with more post-polypectomy bleeding and emergency department visits among anticoagulated patients
}

\author{
Dionne Rebelloa, Mena Bakhit ${ }^{b}$, Thomas R. McCarty, ${ }^{c, d}$, Jason T. Machane, Anil Nagar ${ }^{f, g}$, Steven F. Moss ${ }^{b, h}$ \\ Brown University, Providence, RI; Brigham and Women's Hospital, Boston, MA; Harvard Medical School, Boston, MA; \\ Biostatics and Research, RI Hospital, Providence, RI; Yale University School of Medicine, New Haven, CT; West Haven \\ Veterans Affairs Medical Center, CT; Providence Veteran Affairs Medical Center, RI, USA
}

Abstract

Background Among patients undergoing colonoscopy, anticoagulants are usually stopped and are sometimes substituted by a heparin bridge (hep-bridge). We aimed to assess adverse events associated with hep-bridge compared to temporary cessation of anticoagulants (no-bridge).

Methods This was a single-center, retrospective cohort study that included anticoagulated patients undergoing colonoscopy between 2013 and 2016 at a Veterans Affairs Medical Center. In the nobridge cohort, warfarin was stopped for 5 days and novel anticoagulants for 2 days pre-procedure. In the hep-bridge cohort, anticoagulants were stopped and were substituted by subcutaneous enoxaparin. The primary outcome was post-polypectomy bleeding. Secondary outcomes included cardiovascular events, all-cause adverse events and emergency department or unscheduled ambulatory office visits within 30 days. The predictive values of the HAS-BLED and $\mathrm{CHADS}_{2}$ scores were evaluated.

Results A total of 662 patients were included, of whom 551 underwent polypectomy (mean age 68.6 years; $97.6 \%$ male). Four hundred seventy colonoscopies were performed with no-bridge and 192 with hep-bridge. Post-polypectomy bleeding occurred in $6.0 \%$ of procedures: $5.7 \%$ in the no-bridge cohort compared to $13.0 \%$ of hep-bridge procedures $(\mathrm{P}=0.0038)$. Cardiovascular or thrombotic events occurred after $2.6 \%$ of the no-bridge and $5.2 \%$ of the hep-bridge procedures $(\mathrm{P}=0.1176)$. Emergency department or unscheduled office visits within 30 days were reported after $18.7 \%$ of the no-bridge procedures and $29.7 \%$ of the hep-bridge procedures $(\mathrm{P}<0.0001)$. Neither $\mathrm{CHADS}_{2}$ nor HASBLED scores predicted bleeding.

Conclusion The use of hep-bridge was associated with a greater incidence of post-polypectomy bleeding and more emergency department and unscheduled office visits compared with cessation of all anticoagulants.

Keywords Colonoscopy, bleeding, polypectomy, anticoagulation, bridge therapy, $\mathrm{CHADS}_{2}$

Ann Gastroenterol 2020; 33 (1):1-7

aDepartment of Internal Medicine, Brown University, Providence, RI (Dionne Rebello); 'Division of Gastroenterology, Brown

University, Providence, RI (Mena Bakhit, Steven F. Moss); 'Division of Gastroenterology, Hepatology and Endoscopy, Brigham and Women's Hospital, Boston, MA (Thomas R. McCarty); 'Harvard Medical School,

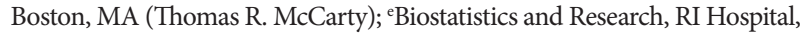
Providence, RI (Jason T. Machan); fSection of Digestive Diseases, Yale University School of Medicine, New Haven, CT (Anil Nagar); ${ }^{\mathrm{T}}$ West Haven Veteran Affairs Medical Center, West Haven, CT (Anil Nagar); ${ }^{\text {PProvidence }}$ Veteran Affairs Medical Center, Providence, RI (Steven F. Moss), USA

Conflict of Interest: None

Correspondence to: Dionne Rebello, MD, Gastroenterology Division, Boston Medical Center, 85 E Concord Street, Boston, MA 02118, USA, e-mail: Dionne.rebello@bmc.org

Received 2 September 2019; accepted 7 October 2019;

published online 1 November 2019

DOI: https://doi.org/10.20524/aog.2019.0430

\section{Introduction}

Colonoscopy remains the preferred method for prevention of colorectal cancer, serving as both a screening and a therapeutic modality. With an estimated 22.4 million colonoscopies performed annually in the United States and another 11 million individuals estimated to be taking anticoagulant therapy, gastroenterologists will increasingly need to be familiar with these medications and understand the associated adverse events [1,2]. It is essential for clinicians to understand, and more importantly reduce, procedure-associated adverse events, including the most common post-polypectomy bleeding $[3,4]$. Reported to occur in approximately $0.3-10 \%$ of cases overall, the incidence of post-polypectomy bleeding related specifically to a population of patients on anticoagulants is less well defined $[4,5]$. 
Patients using anticoagulation therapy present a unique and common clinical dilemma for the gastroenterologist: balancing the risk of anticoagulation cessation against the risk of procedure-associated hemorrhage [6]. Additional concerns that must be factored into this decision include the risk of thromboembolic events, reported to be as high as $3 \%$ when anticoagulation is withheld $[7,8]$. To reduce this risk of thromboembolic events, high-risk patients have traditionally been switched from warfarin to a shorter-acting bridge anticoagulation therapy (i.e., unfractionated or lowmolecular-weight heparin [LMWH]) $[4,6,9]$. Despite current guidelines from national societies regarding the management of periprocedural anticoagulants, it remains unclear whether stopping anticoagulants temporarily and substituting them with LMWH as a temporary anticoagulant bridge therapy will result in more post-colonoscopy bleeding and a smaller number of thromboembolic events compared to complete anticoagulant interruption [10-12].

Thus, the aim of this study was to evaluate both the bleeding adverse events and thromboembolic events in patients undergoing colonoscopy who usually take anticoagulants, comparing those using a heparin bridge (hep-bridge) with those who temporarily stop all anticoagulation medications.

\section{Patients and methods}

\section{Study design}

This was a single-center, retrospective cohort study conducted at the Providence Veterans Affairs (VA) Medical Center in Rhode Island, using data extracted from the Veterans Affairs Computerized Patient Record System (CPRS). The study was approved by the Institutional Review Board at the Providence VA Medical Center on December 1, 2016. All patients who underwent outpatient and inpatient colonoscopy between January 2013 and November 2016 and had been prescribed anticoagulation therapy at the time of the procedure were identified. A systematic chart review was then performed to collect the relevant data, including demographics, periprocedural anticoagulation strategy and postprocedural outcomes. Colonoscopies were performed either by a trainee under the direct supervision of an experienced, board-certified gastroenterologist, or by the gastroenterologist themselves. Multiple colonoscopies in the same patient were included only if they occurred more than one year apart.

\section{Cohort selection and anticoagulation strategy}

Patient records were reviewed and categorized into 2 specific cohorts: a temporary anticoagulation cessation group ("no-bridge") and a bridging group ("hep-bridge"). The choice of periprocedural anticoagulation strategy was based upon patient-specific risk factors and was made by a clinical pharmacist in the hospital's anti-coagulation clinic, where all patients were managed using a standardized Providence VA protocol. In this protocol, patients with atrial fibrillation and $\mathrm{CHADS}_{2}$ scores of 1 to 2 were not prescribed hep-bridge $[13,14]$. Patients with atrial fibrillation and $\mathrm{CHADS}_{2}$ scores of 3 to 4 were not prescribed a bridge unless the patient had a history of cerebrovascular accident. For those with atrial fibrillation and a $\mathrm{CHADS}_{2}$ score of 5 to 6 , hep-bridge was prescribed. Additionally, any patient with a mechanical cardiac valve was prescribed hep-bridge.

For patients in the no-bridge cohort, warfarin was stopped 5 days pre-procedure and reinitiated immediately following colonoscopy, as per standard protocol. In the hep-bridge cohort, warfarin was held and subcutaneous enoxaparin (1 mg/ $\mathrm{kg}$ b.i.d.) was started 5 days prior to the procedure. Enoxaparin was stopped on the day of the colonoscopy and warfarin was restarted immediately post-procedure and continued until the target international normalized ratio (INR) was reached. Enoxaparin was typically continued with warfarin for 5 days post-procedure in the hep-bridge cohort. Other oral anticoagulants (apixaban, rivaroxaban) were stopped 2 days pre-procedure, without any hep-bridge, and reinitiated immediately following colonoscopy, as per hospital protocol.

\section{Clinical characteristics and covariates}

Demographic information including sex, age, and race/ethnicity was recorded. Additional patient-specific characteristics, including specific risk factors for bleeding (i.e. end-stage renal disease or cirrhosis), type of anticoagulant and indication for use, platelet count at time of procedure and INR, were also documented. $\mathrm{CHADS}_{2}$ and HAS-BLED scores were calculated regardless of the indication for anticoagulation, not only in patients with documented atrial fibrillation [13-16]. Procedure-related measures collected included indication for colonoscopy, whether polypectomy was performed, method of endoscopic polyp resection (cold/hot biopsy or cold/hot snare), polyp location, size of largest polyp, number of polyps identified, and whether placement of an endoscopic clip was performed. These data were collected based on previous reports that documented factors associated with post-polypectomy bleeding [5,17-19].

\section{Adverse events and post-polypectomy bleeding definition}

Any complication occurring within 30 days after the procedure was determined by chart review Immediate procedural bleeding was included if an additional intervention such as a clip was required during the procedure for hemostasis. Meaningful delayed post-procedural bleeding was defined as either rectal bleeding on gross examination of stool that led the patient to visit the physician or emergency department, or direct visualization on repeat colonoscopy within 30 days of the initial procedure. The severity of gastrointestinal bleeding was determined by the need for transfusion. Non-gastrointestinal related adverse events included any confirmed thromboembolic event (deep venous thrombosis, pulmonary embolism, 
myocardial ischemia/infarction, cerebrovascular accident, peripheral arterial thrombosis, and any non-gastrointestinal complication that resulted in an emergency department visit, hospital admission, or unscheduled outpatient primary care visit within 30 days of the colonoscopy. The records of such events were reviewed to determine any possible relationship to colonoscopy, anticoagulation, or thromboembolic etiology.

\section{Measured outcomes}

The primary outcome measure in this study was the incidence of post-polypectomy bleeding. Secondary outcomes included cardiovascular events, defined as thrombotic events, all-cause adverse events, emergency department or unscheduled ambulatory office visits within 30 days of the procedure, and predictive values of the $\mathrm{CHADS}_{2}$ and HASBLED scores for adverse events.

\section{Statistical analysis}

Patient characteristics and outcomes were compared using univariate $t$-tests for continuous variables. SAS version 9.4 (The SAS Institute, Cary, NC) was used for multiple regression analyses. Multiple regression was used to model the probabilities of dichotomous outcomes as a function of bleed risk severity $\left(\mathrm{CHADS}_{2}\right.$ or HAS-BLED) and whether or not a bridge was used, along with their interaction. Alpha per comparison was set to 0.05 (i.e. without adjustment) owing to the likely correlated nature of the multiple hypotheses being tested and a desire to be liberal in detecting a differential risk for clinical reasons. Classical sandwich estimation was used to adjust for any model misspecification.

\section{Results}

\section{Demographic and clinical characteristics}

A total of 662 colonoscopy procedures in 610 patients were identified during the predetermined study period, based on the above inclusion and exclusion criteria. Among these 662 procedures, 470 procedures were documented to have been performed without bridge anticoagulation and 192 procedures with enoxaparin bridge therapy. The mean age of the patients was 68.6 years and $97.6 \%$ were male. The most common indication for anticoagulation was atrial fibrillation or atrial flutter, noted in 341 procedures (51.5\%), followed by a history of deep venous thrombosis or pulmonary embolism, in 204 procedures (30.8\%) (Fig. 1). The most common indication for colonoscopy was polyp surveillance in 369 patients (55.7\%), followed by colorectal cancer screening in 137 patients (20.7\%) (Fig. 2). Polypectomy was performed in a total of 551 procedures (83.2\%) and included 498 cold biopsy polypectomies and 87 snare polypectomies (Fig. 3). Additional

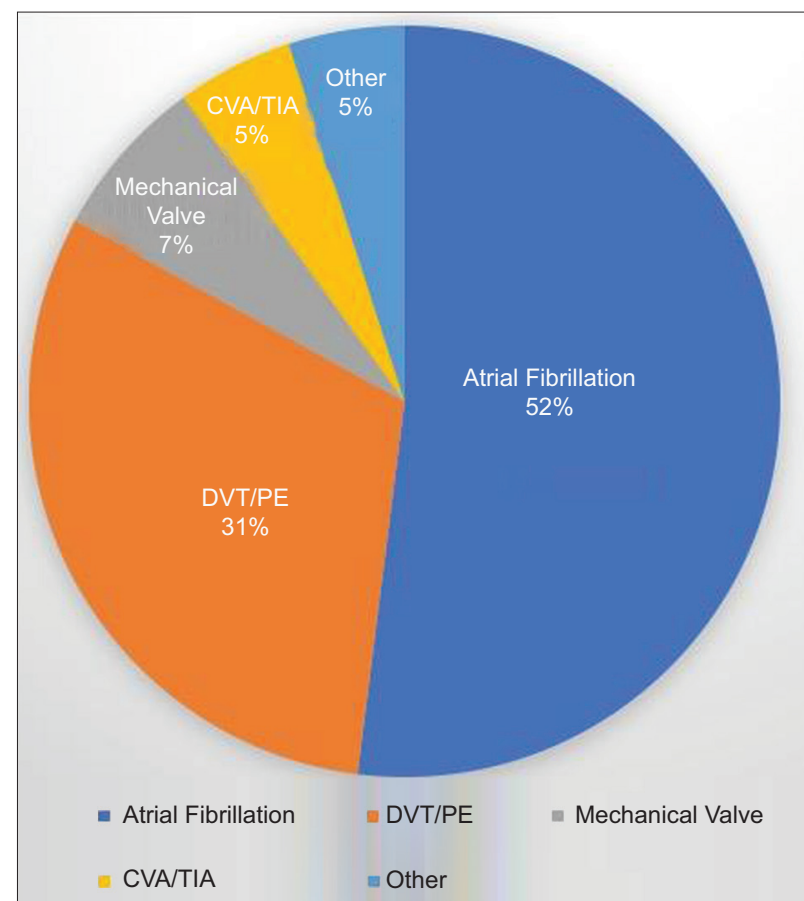

Figure 1 Indications for anticoagulation among veterans CVA/TIA, cerebrovascular accident/transient ischemic attack; DVT/PE, deep vein thrombosis/pulmonary embolism

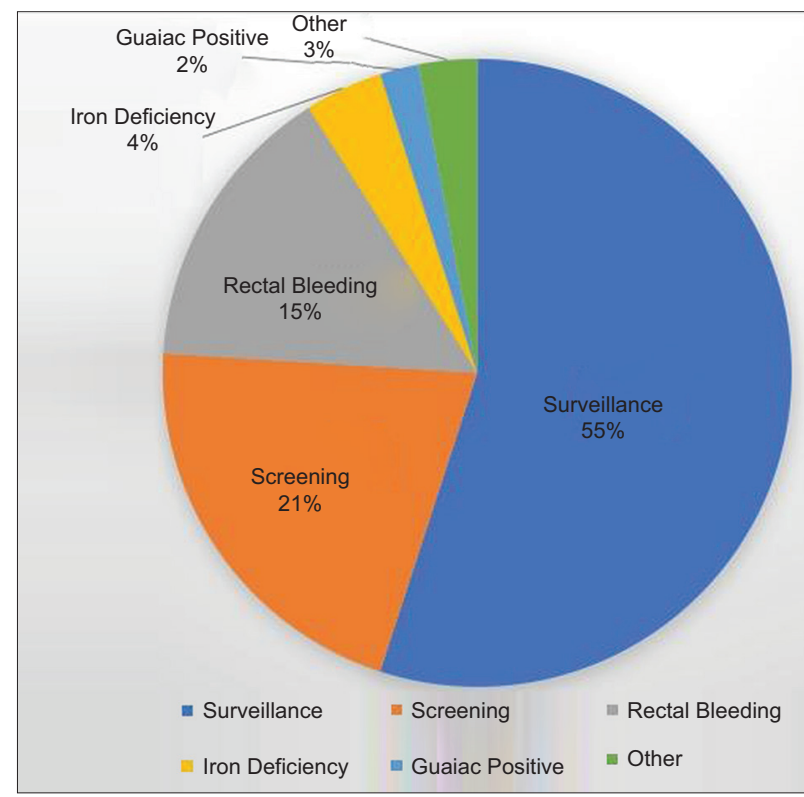

Figure 2 Indications for colonoscopy among veterans

demographic information, including patient and procedure characteristics, is summarized in Table 1.

Post-polypectomy bleeding was observed in $7.85 \%$ of total procedures $(n=52)$. Subdividing this by cohort, there was a statistically significant difference in post-polypectomy bleeding: $5.7 \%$ of non-bridge procedures $(n=27)$ and $13.0 \%$ $(\mathrm{n}=25)$ of hep-bridge procedures $(\mathrm{P}=0.0038)$. When the data were further stratified according to polypectomy method, 
there was no significant difference in post-polypectomy bleeding between non-bridge and hep-bridge groups, whether snare cautery-74\% $(\mathrm{n}=20)$ vs. $80 \%(\mathrm{n}=20)$, respectively; $\mathrm{P}=0.8549$ —or cold biopsy- $1.5 \%(\mathrm{n}=7)$ vs. $2.6 \%$, respectively $(\mathrm{n}=5) ; \mathrm{P}=0.6123$ - was used. Adverse cardiovascular or thromboembolic events occurred in $3.9 \%$ of the total

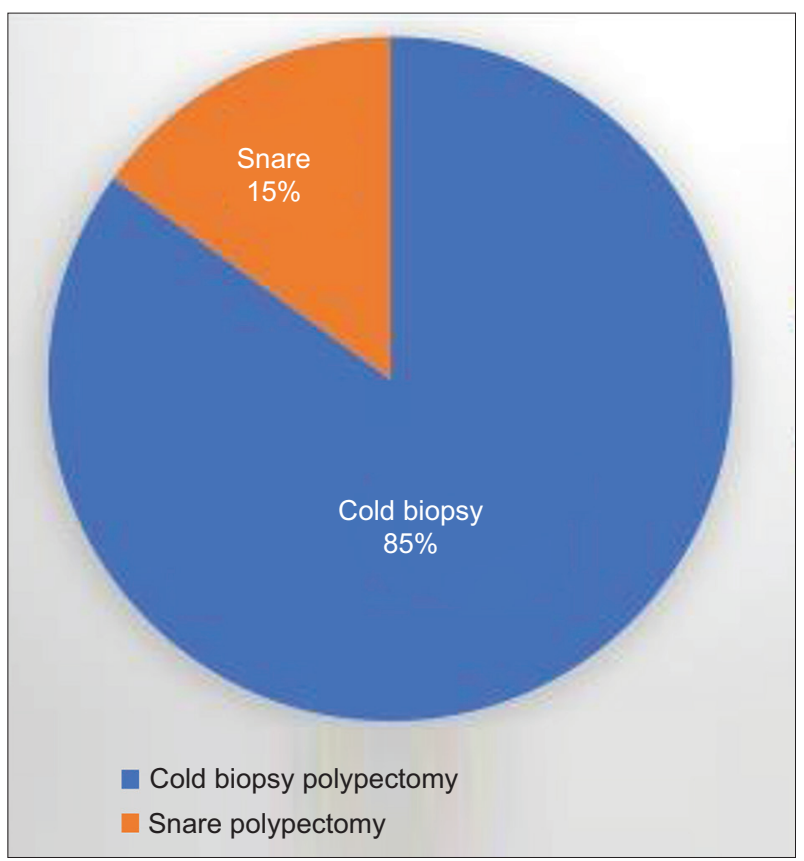

Figure 3 Type of polypectomy method during colonoscopy procedures ( $n=26): 2.6 \%$ of the no-bridge and $5.2 \%$ of the hepbridge procedures $(\mathrm{P}=0.1176)$. The incidence of post-procedure hospitalization, and emergency department and unscheduled ambulatory visits differed significantly between the 2 groups: 88 non-bridge procedures $(18.7 \%)$ and 57 bridge procedures (29.7\%) $\quad(\mathrm{P}<0.0001)$. The most common chief complaints associated with these unscheduled emergency department and ambulatory visits were orthostatic ( $n=19$ for non-bridge, $n=11$ for hep-bridge), urinary ( $n=9$ for non-bridge, $n=4$ for hepbridge), or musculoskeletal symptoms ( $\mathrm{n}=4$ for non-bridge, $\mathrm{n}=9$ for hep-bridge). Thirty-day outcomes post-colonoscopy are shown in Table 2.

\section{Multivariate analysis}

Multivariate logistic regression was also performed to evaluate $\mathrm{CHADS}_{2}$ and HAS-BLED scores as predictors of 30-day unscheduled visits and gastrointestinal adverse events. Neither $\mathrm{CHADS}_{2}$ nor HAS-BLED scores were predictive of adverse events.

\section{Discussion}

As the frequency and utilization of both colonoscopies and anticoagulation continues to rise, endoscopists will encounter an increasing number of patients taking anticoagulant medications $[1,20]$. Therefore, examination of these therapeutic

Table 1 Baseline characteristics of veterans who received heparin bridge compared to those in whom anticoagulation was interrupted

\begin{tabular}{|c|c|c|c|}
\hline Baseline characteristics & No-bridge cohort $(n=470)$ & Bridge cohort $(n=192)$ & P-value \\
\hline Mean age (years) & 69.3 & 67.1 & $<0.0001$ \\
\hline $\begin{array}{l}\text { Race/Ethnicity } \\
\text { Caucasian } \\
\text { Other }\end{array}$ & $\begin{array}{c}444(94.5 \%) \\
26(5.5 \%)\end{array}$ & $\begin{array}{c}187(97.4 \%) \\
5(2.6 \%)\end{array}$ & 0.1032 \\
\hline $\begin{array}{l}\text { Type of anticoagulation } \\
\text { Warfarin } \\
\text { Direct-acting oral anticoagulant }\end{array}$ & $\begin{array}{c}401(85.3 \%) \\
69(4.7 \%)\end{array}$ & $\begin{array}{c}183(95.3 \%) \\
9(4.7 \%)\end{array}$ & 0.0003 \\
\hline Cold biopsy polypectomy & $400(85.1 \%)$ & $151(78.6 \%)$ & 0.5383 \\
\hline Clip placement required & $95(20.2 \%)$ & $29(15.1 \%)$ & 0.2017 \\
\hline
\end{tabular}

Table 2 Thirty-day outcomes among veterans with heparin bridge therapy compared to those in whom anticoagulation was interrupted

\begin{tabular}{lccc}
\hline 30-Day adverse events & No-bridge cohort $(\mathrm{n}=470)$ & Bridge cohort $(\mathrm{n}=192)$ & P-value \\
\hline Post-polypectomy bleeding & $27(5.7 \%)$ & $25(13.0 \%)$ & 0.0038 \\
Blood transfusion required & $9(1.9 \%)$ & $3(1.6 \%)$ & 0.7618 \\
Emergency department or ambulatory visit & $88(18.7 \%)$ & $57(29.7 \%)$ & $<0.0001$ \\
Cardiovascular or thromboembolic event & $12(2.6 \%)$ & $10(5.2 \%)$ & 0.1176 \\
\hline
\end{tabular}


agents and associated management decisions-whether to stop anticoagulant therapy entirely or use hep-bridge-remain critical for improving patient outcomes and reducing overall healthcare-related costs. The results of this study demonstrated that continued anticoagulation (i.e. hep-bridge therapy) is associated with higher rates of post-polypectomy bleeding, as well as other periprocedural adverse events, compared with temporary anticoagulation cessation.

Antithrombotic therapy has been proven to reduce the risk of thromboembolic events in patients at high risk for this complication, but it increases the risk of post-polypectomy bleeding in patients undergoing colonoscopy [4,5,18,21]. Because the temporary cessation of warfarin increases the risk of thromboembolic events, hep-bridge therapy is often prescribed as a compromise between the competing health risks of bleeding after polypectomy and precipitation of a potentially life-threatening thromboembolic event $[4,6,10]$. Our study provides real-world estimates of the rates of postpolypectomy bleeding and non-gastrointestinal adverse events following colonoscopy and polypectomy in patients who discontinue their anticoagulants completely versus those who receive a hep-bridge.

Given these findings, it is also important to understand the mechanism of anticoagulation and relation to post-polypectomy bleeding. The ability to form a clot post-polypectomy relies on primary and secondary hemostasis. Unlike aspirin and other anti-platelet agents, such as clopidogrel, the use of hepbridge will inhibit the formation of insoluble, cross-linked fibrin and the activation of thrombin. Interestingly, previous studies have also shown anticoagulants to be associated with a higher risk of post-polypectomy bleeding, but not in direct relation to antiplatelet agents, perhaps because of the impact on secondary, rather than primary hemostasis [22-26]. Postpolypectomy bleeding was observed in $7.85 \%$ of cases in this study $(5.7 \%$ of non-bridge procedures and $13.0 \%$ of bridging procedures). While initially these rates appear to be higher than those previously reported (bleeding in approximately 1-2\% of post-polypectomy cases), other studies have demonstrated delayed bleeding rates as high as $34 \%$ among patients with anticoagulant use [18,21,27,28].

Many risk factors have been associated with postpolypectomy bleeding, including age greater than 75 years, polyp size greater than $1 \mathrm{~cm}$, location and morphology of the polyp, resection technique, and notably anticoagulation, with a 3- to 5-fold greater incidence of procedure-related bleeding in patients taking anticoagulants [4-6,17-19]. Rates of thromboembolic events in the setting of temporary cessation of anticoagulation for endoscopy have been reported to be as high as $3 \%$, with an absolute risk of $1 \%$ for patients who interrupt anticoagulation for $\leq 5$ days $[7,8]$. Guidelines from the American College of Chest Physicians regarding anticoagulation management in patients with non-valvular atrial fibrillation in the periprocedural period recommend considering stopping vitamin $\mathrm{K}$ antagonists, based on the risk of thromboembolism and bleeding, and assessing CHADS, scores to determine the need for bridge therapy [11].

Current guidelines from both the European and American Gastroenterology Associations regarding the management of anticoagulation and the use of bridge therapy, specifically in high-risk patients undergoing endoscopy, are largely based on low-quality evidence and expert opinion $[6,12]$. The guidelines from the British Society of Gastroenterology and European Society of Gastrointestinal Endoscopy classify colonoscopy with polypectomy as a high-risk procedure for post-procedural bleeding, and suggest that pre-procedural bridge therapy should be used for patients on warfarin who are at high risk for thromboembolic events. These high-risk patients include those with atrial fibrillation and mitral stenosis, patients with a prosthetic metal heart valve in the mitral position, a prosthetic heart valve and atrial fibrillation, and patients within 3 months of a venous thromboembolism. The American Society for Gastrointestinal Endoscopy specifically recommends bridge therapy for high-risk patients with atrial fibrillation on warfarin who have mechanical valves, a history of a stroke, and a $\mathrm{CHADS}_{2}$ score $\geq 2$. The Society also recommends bridge therapy for patients with a mechanical aortic valve, any thromboembolic risk factor, and older-generation and mechanical mitral valve replacements in those with valvular heart disease [6]. However, these societal guidelines from experts are based on very limited data. Previously published studies have compared bridge therapy to temporary cessation of anticoagulation with regard to post-polypectomy bleeding; however, they generally comprised small numbers of patients, while in one of them important thromboembolic event outcomes were not reported $[23,29]$.

A retrospective study showed a high incidence of postpolypectomy bleeding with hep-bridge therapy $(8.3 \%)$ for antiplatelet agents, compared with an even higher $24.2 \%$ with hep-bridge therapy for warfarin [23]. Similar results were noted in a large meta-analysis, which reported that patients receiving periprocedural hep-bridge were at increased risk of overall bleeding ( $>5$-fold increased risk) and major bleeding ( $>3$-fold increased risk), whereas the risk of thromboembolic events was similar to that of non-bridged patients [24]. A prospective study evaluated the incidence of gastrointestinal bleeding in patients on warfarin or hep-bridge therapy and found that the use of enoxaparin therapy was not significantly associated with a risk of postprocedural hemorrhage [30]. Likewise, another study concluded that hep-bridge therapy was associated with a low risk of thromboembolic and major bleeding events in highrisk patients who required temporary interruption of warfarin before undergoing nonsurgical invasive procedures, such as gastrointestinal endoscopy or cardiac catheterization [31]. In one randomized controlled trial comparing bridge therapy to a no-bridge strategy in patients undergoing a variety of elective surgical and other invasive procedures, the use of hep-bridge resulted in more major bleeding, without a significant benefit in terms of preventing arterial thrombosis [32].

In our study, the use of hep-bridge was associated with a greater incidence of gastrointestinal bleeding, as well as more visits to the emergency department and other unscheduled ambulatory office visits within 30 days of the colonoscopy. The most common complaints prompting these visits were orthostatic symptoms, urinary complaints or musculoskeletal symptoms, though these symptoms cannot be easily attributed to the hep-bridge alone. Moreover, the HAS-BLED score had 


\section{Summary Box}

\section{What is already known:}

- Colonoscopies in patients receiving anticoagulation therapy have become increasingly common for gastroenterologists

- There is a need to balance the risk of anticoagulation cessation against the risk of procedure-associated hemorrhage so as to make these procedures as safe and effective as possible

- At the time of colonoscopy, anticoagulants may be stopped and substituted by a heparin bridge (hepbridge), although there are limited data describing the adverse events of this practice

\section{What the new findings are:}

- Among a population of veterans, the use of hepbridge was associated with a greater incidence of post-polypectomy bleeding

- The hep-bridge strategy was associated with more emergency department and unscheduled office visits compared to the cessation of all anticoagulants

no predictive value for such visits. In addition, the predictive value of this score for post-polypectomy bleeding risk appears to be inconsistent. In a recent prospective, observational, multi-center registry study of patients with a history of venous thromboembolism who started anticoagulation with a HASBLED score of $\geq 3$, the score had good specificity and negative predictive value but lacked sensitivity when predicting bleeding events in the first 6 months of anticoagulation therapy [33].

Our study is one of the largest studies in the United States to evaluate the use of bridge therapy exclusively in the setting of colonoscopy, the most common endoscopic procedure performed in the United States. Since our patients were all from a VA hospital, with a relatively homogenous, largely male and Caucasian population and with a high frequency of polypectomy, the results may not be generalizable to other populations. Furthermore, there was a significant difference in age between the 2 groups, suggesting that the 2 cohorts may not be totally comparable. Another limitation is that our study was not controlled, but rather a single-center retrospective analysis of current practice. In this study, patients with atrial fibrillation and $\mathrm{CHADS}_{2}$ scores of 1 to 4 were not prescribed hep-bridge, in accordance with our VA protocol. For those with atrial fibrillation and a $\mathrm{CHADS}_{2}$ score of 5 to 6 , hepbridge therapy was prescribed. It is possible some patients may have developed rebleeding and presented to another institution or were lost to follow up so that any events were not recorded.

In conclusion, our results clearly indicate that hep-bridge, prescribed to patients with the highest risk of cardiovascular morbidity, is nevertheless associated with higher rates of both post-polypectomy bleeding and other periprocedural adverse events compared to the temporary cessation of anticoagulation. Since neither the $\mathrm{CHADS}_{2}$ nor the HAS-BLED scores was predictive of adverse events, 30-day unscheduled visits or 30-day hospital admissions, this suggests that there is a need for a better scoring system that will enable practitioners to estimate the likelihood of adverse events after colonoscopy in anticoagulated patients, whether or not they receive hepbridge.

\section{Acknowledgment}

We would like to thank Margaret Quillin, PharmD, of the Providence VA Medical Center, for assistance with the acquisition of data.

\section{References}

1. Barnes GD, Lucas E, Alexander GC, Goldberger ZD. National trends in ambulatory oral anticoagulant use. Am J Med 2015;128:1300-1305.e2.

2. Joseph DA, Meester RG, Zauber AG, et al. Colorectal cancer screening: Estimated future colonoscopy need and current volume and capacity. Cancer 2016;122:2479-2486.

3. Rex DK, Lewis BS, Waye JD. Colonoscopy and endoscopic therapy for delayed post-polypectomy hemorrhage. Gastrointest Endosc 1992;38:127-129.

4. Anderson MA, Ben-Menachem T, Gan SI, et al; ASGE Standards of Practice Committee. Management of antithrombotic agents for endoscopic procedures. Gastrointest Endosc 2009;70:1060-1070.

5. Boustière C, Veitch A, Vanbiervliet G, et al; European Society of Gastrointestinal Endoscopy. Endoscopy and antiplatelet agents. European Society of Gastrointestinal Endoscopy (ESGE) Guideline. Endoscopy 2011;43:445-461.

6. Acosta RD, Abraham NS, Chandrasekhara V, et al; ASGE Standards of Practice Committee. The management of antithrombotic agents for patients undergoing GI endoscopy. Gastrointest Endosc 2016;83:3-16.

7. Blacker DJ, Wijdicks EF, McClelland RL. Stroke risk in anticoagulated patients with atrial fibrillation undergoing endoscopy. Neurology 2003;61:964-968.

8. Garcia DA, Regan S, Henault LE, et al. Risk of thromboembolism with short-term interruption of warfarin therapy. Arch Intern Med 2008; 168:63-69.

9. Fuster V, Rydén LE, Cannom DS, et al; Heart Rhythm Society. ACC/ AHA/ESC 2006 Guidelines for the Management of Patients with Atrial Fibrillation: a report of the American College of Cardiology/ American Heart Association Task Force on Practice Guidelines and the European Society of Cardiology Committee for Practice Guidelines (Writing Committee to Revise the 2001 Guidelines for the Management of Patients With Atrial Fibrillation): developed in collaboration with the European Heart Rhythm Association and the Heart Rhythm Society. Circulation 2006;114:e257-e354.

10. Doherty JU, Gluckman TJ, Hucker WJ, et al. 2017 ACC expert consensus decision pathway for periprocedural management of anticoagulation in patients with nonvalvular atrial fibrillation: a report of the American College of Cardiology Clinical 
Expert Consensus Document Task Force. J Am Coll Cardiol 2017;69:871-898.

11. Douketis JD, Spyropoulos AC, Spencer FA, et al. Perioperative management of antithrombotic therapy: antithrombotic therapy and prevention of thrombosis, $9^{\text {th }}$ ed: American College of Chest Physicians Evidence-Based Clinical Practice Guidelines. Chest 2012;141:e326S-e350S.

12. Veitch AM, Vanbiervliet G, Gershlick AH, et al. Endoscopy in patients on antiplatelet or anticoagulant therapy, including direct oral anticoagulants: British Society of Gastroenterology (BSG) and European Society of Gastrointestinal Endoscopy (ESGE) guidelines. Gut 2016;65:374-389.

13. Gage BF, van Walraven C, Pearce L, et al. Selecting patients with atrial fibrillation for anticoagulation: stroke risk stratification in patients taking aspirin. Circulation 2004;110:2287-2292.

14. Gage BF, Waterman AD, Shannon W, Boechler M, Rich MW, Radford MJ. Validation of clinical classification schemes for predicting stroke: results from the National Registry of Atrial Fibrillation. JAMA 2001;285:2864-2870.

15. Lip GY, Frison L, Halperin JL, Lane DA. Comparative validation of a novel risk score for predicting bleeding risk in anticoagulated patients with atrial fibrillation: the HAS-BLED (Hypertension, Abnormal Renal/Liver Function, Stroke, Bleeding History or Predisposition, Labile INR, Elderly, Drugs/Alcohol Concomitantly) score. J Am Coll Cardiol 2011;57:173-180.

16. Pisters R, Lane DA, Nieuwlaat R, de Vos CB, Crijns HJ, Lip GY. A novel user-friendly score (HAS-BLED) to assess 1-year risk of major bleeding in patients with atrial fibrillation: the Euro Heart Survey. Chest 2010;138:1093-1100.

17. Kim HS, Kim TI, Kim WH, et al. Risk factors for immediate postpolypectomy bleeding of the colon: a multicenter study. Am J Gastroenterol 2006;101:1333-1341.

18. Sawhney MS, Salfiti N, Nelson DB, Lederle FA, Bond JH. Risk factors for severe delayed postpolypectomy bleeding. Endoscopy 2008;40:115-119.

19. Watabe H, Yamaji Y, Okamoto M, et al. Risk assessment for delayed hemorrhagic complication of colonic polypectomy: polyprelated factors and patient-related factors. Gastrointest Endosc 2006;64:73-78.

20. Kirley K, Qato DM, Kornfield R, Stafford RS, Alexander GC. National trends in oral anticoagulant use in the United States, 2007 to 2011. Circ Cardiovasc Qual Outcomes 2012;5:615-621.

21. Rabeneck L, Paszat LF, Hilsden RJ, et al. Bleeding and perforation after outpatient colonoscopy and their risk factors in usual clinical practice. Gastroenterology 2008;135:1899-1906.

22. Beppu K, Osada T, Sakamoto N, et al. Optimal timing for resuming antithrombotic agents and risk factors for delayed bleeding after endoscopic resection of colorectal tumors. Gastroenterol Res Pract 2014;2014:825179.

23. Inoue T, Nishida T, Maekawa A, et al. Clinical features of postpolypectomy bleeding associated with heparin bridge therapy. Dig Endosc 2014;26:243-249.

24. Siegal D, Yudin J, Kaatz S, Douketis JD, Lim W, Spyropoulos AC. Periprocedural heparin bridging in patients receiving vitamin $\mathrm{K}$ antagonists: systematic review and meta-analysis of bleeding and thromboembolic rates. Circulation 2012;126:1630-1639.

25. Ishigami $H$, Arai $M$, Matsumura $T$, et al. Heparin-bridging therapy is associated with a high risk of post-polypectomy bleeding regardless of polyp size. Dig Endosc 2017;29:65-72.

26. Radaelli F, Fuccio L, Paggi S, et al; Bowell Group. Periendoscopic management of direct oral anticoagulants: a prospective cohort study. Gut 2019;68:969-976.

27. Waye JD, Lewis BS, Yessayan S. Colonoscopy: a prospective report of complications. J Clin Gastroenterol 1992;15:347-351.

28. Rutter MD, Nickerson C, Rees CJ, Patnick J, Blanks RG. Risk factors for adverse events related to polypectomy in the English Bowel Cancer Screening Programme. Endoscopy 2014;46:90-97.

29. Constans M, Santamaria A, Mateo J, Pujol N, Souto JC, Fontcuberta J. Low-molecular-weight heparin as bridging therapy during interruption of oral anticoagulation in patients undergoing colonoscopy or gastroscopy. Int J Clin Pract 2007;61:212-217.

30. Gerson LB, Michaels L, Ullah N, Gage B, Williams L. Adverse events associated with anticoagulation therapy in the periendoscopic period. Gastrointest Endosc 2010;71:1211-1217.

31. Douketis JD, Johnson JA, Turpie AG. Low-molecular-weight heparin as bridging anticoagulation during interruption of warfarin: assessment of a standardized periprocedural anticoagulation regimen. Arch Intern Med 2004;164:1319-1326.

32. Douketis JD, Spyropoulos AC, Kaatz S, et al; BRIDGE Investigators. Perioperative bridging anticoagulation in patients with atrial fibrillation. N Engl J Med 2015;373:823-833.

33. Omran H, Bauersachs R, Rübenacker S, Goss F, Hammerstingl C. The HAS-BLED score predicts bleedings during bridging of chronic oral anticoagulation. Results from the national multicentre BNK Online bRiDging REgistRy (BORDER). Thromb Haemost 2012;108:65-73. 